\title{
Two Non-steroidal Anti-inflammatory Drugs, Niflumic Acid and Diclofenac, Inhibit the Human Glutamate Transporter EAAT1 Through Different Mechanisms
}

\author{
Kanako Takahashi ${ }^{1,2}$, Reiko Ishii-Nozawa ${ }^{1}$, Kouichi Takeuchi ${ }^{1}$, Ken Nakazawa ${ }^{2}$, and Kaoru Sato ${ }^{2, *}$ \\ ${ }^{I}$ Department of Clinical Pharmacology, Meiji Pharmaceutical University, Noshio 2-522-1, Kiyose, Tokyo 204-8588, Japan \\ ${ }^{2}$ Division of Pharmacology, National Institute of Health Sciences, 1-18-1 Kamiyoga, Setagaya, Tokyo 158-8501, Japan
}

Received September 17, 2009; Accepted November 14, 2009

\begin{abstract}
We investigated the effects of non-steroidal anti-inflammatory drugs on substrateinduced currents of L-glutamate (L-Glu) transporter EAAT1 expressed in Xenopus laevis oocytes. Niflumic acid (NFA) and diclofenac inhibited L-Glu-induced current through EAAT1 in a noncompetitive manner. NFA produced a leftward shift in reversal potential $\left(\mathrm{E}_{\text {rev }}\right)$ of L-Glu-induced current and increased current amplitude at the potentials more negative than $-100 \mathrm{mV}$. Diclofenac had no effects on $\mathrm{E}_{\mathrm{rev}}$ and inhibited the current amplitude to the same extent at all negative potentials. These results indicate that NFA and diclofenac inhibit the L-Glu-induced EAAT1 current via different mechanisms.
\end{abstract}

[Supplementary methods and Figure: available only at http://dx.doi.org/10.1254/jphs.09260SC]

Keywords: L-glutamate transporter, niflumic acid, diclofenac

L-Glutamate (L-Glu) transporters, EAATs, are the only significant mechanism for removal of L-Glu from extracellular fluid and maintenance of non-toxic concentrations. A growing body of evidence has suggested the correlation of EAATs with synaptic transmission and a variety of central nervous system (CNS) diseases (1).

Non-steroidal anti-inflammatory drugs (NSAIDs) are major anti-inflammation drugs and their effects are attributed to the inhibition of cyclooxygenase. Although NSAIDs are reported to have diverse effects on the CNS $(2,3)$, their effects cannot be explained only by their anti-inflammatory effects.

In recent report, fenamates, a group of NSAIDs, modulated substrate-induced current through EAATs $(4,5)$, suggesting a new molecular target for NSAIDs. We therefore investigated the effects of other types of NSAIDs on EAAT1.

All procedures were in accordance with the Guiding Principles for the Care and Use of Laboratory Animals approved by The Japanese Pharmacological Society. The detailed methods for expression of EAAT1 in oocytes

*Corresponding author. kasato@nihs.go.jp

Published online in J-STAGE on January 6, 2010 (in advance)

doi: 10.1254/jphs.09260SC and electrophysiology were shown in the Supplementary Methods (available in the online version only). L-Glu was applied for $15 \mathrm{~s}$ with regular 30-s intervals. NSAIDs were applied from $30 \mathrm{~s}$ before to $5 \mathrm{~s}$ after the end of the application of L-Glu.

The NSAIDs were dissolved as follows: niflumic acid (NFA) (Sigma, St. Louis, MO, USA), $300 \mathrm{mM}$ in DMSO; diclofenac (Wako, Osaka), $300 \mathrm{mM}$ in $\mathrm{MeOH}$; aspirin (Wako), $300 \mathrm{mM}$ in $\mathrm{EtOH}$; and indomethacin (Sigma), $100 \mathrm{mM}$ in EtOH. Arachidonic acid (AA) (Calbiochem, Darmstadt, Germany) was dissolved at 100 $\mathrm{mM}$ in DMSO.

All data were given as the mean \pm S.E.M. $P$ values were obtained by statistical analysis, as noted in the figure legends.

The left traces in Fig. 1A-a illustrate inward control current produced by L-Glu $(30 \mu \mathrm{M})$ in Xenopus oocytes expressing EAAT1 at $-50 \mathrm{mV}$ (bold line) and -120 $\mathrm{mV}$ (thin line). We examined the effects of a variety of NSAIDs (Fig. 1: A and B). At $-50 \mathrm{mV}$, NFA $(300 \mu \mathrm{M}-3$ $\mathrm{mM}$ ) inhibited the EAAT1 current dose-dependently. At $-120 \mathrm{mV}$, NFA enhanced EAAT1 current and the effect was significant at $3 \mathrm{mM}$. Xenopus oocytes have endogenously $\mathrm{Ca}^{2+}$-activated $\mathrm{Cl}^{-}$channels $(\mathrm{CaCC})$ and $\mathrm{Ca}^{2+}$ entry through voltage-dependent $\mathrm{Ca}^{2+}$ channels elicits a 
A
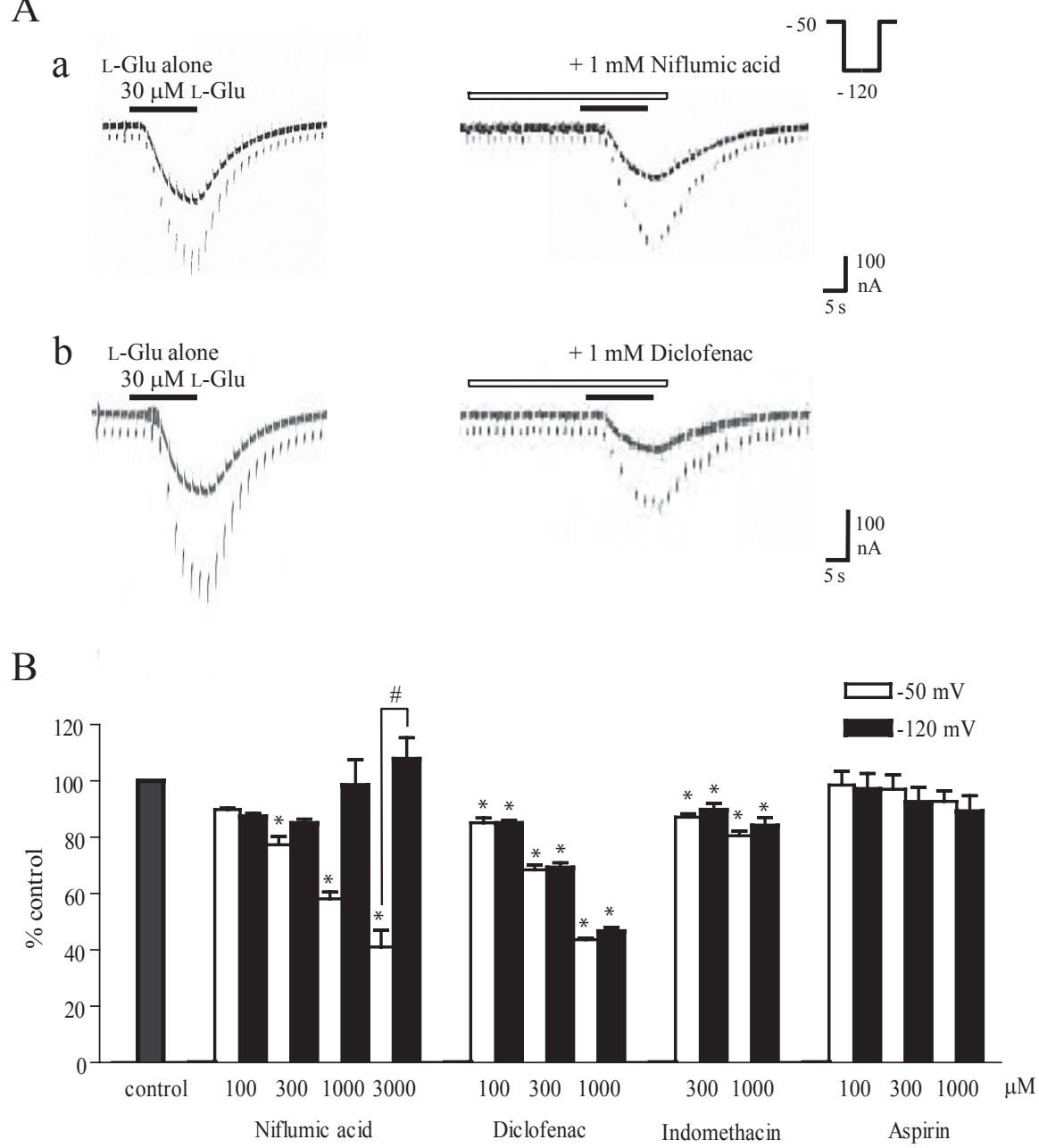

$\mathrm{C}$

$-50 \mathrm{mV}$
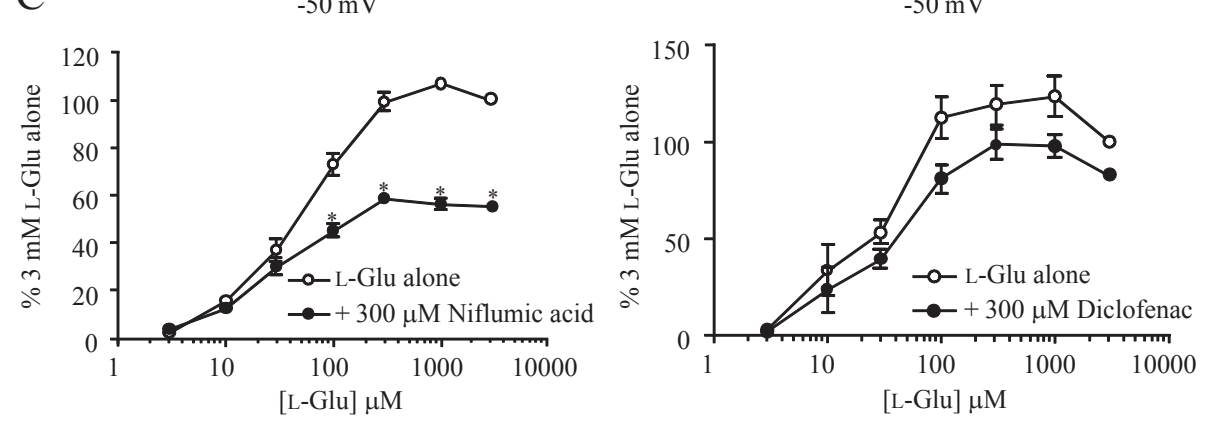

Fig. 1. Effects of NFA and diclofenac on L-Glu-induced currents in EAAT1-expressing Xenopus oocytes. A: The traces of L-Glu (30 $\mu \mathrm{M}$ )-induced inward currents in the presence or the absence of NFA (a) or diclofenac (b) at $-50 \mathrm{mV}$ (bold line) and $-120 \mathrm{mV}$ (thin line). The oocytes were held at $-50 \mathrm{mV}$ and hyperpolarized to $-120 \mathrm{mV}$ for $400 \mathrm{~ms}$, every $2 \mathrm{~s}$. B: Concentration-response relationships for various NSAIDs at -50 and $-120 \mathrm{mV}$. The amplitude of EAAT1 current in the presence of the drug was normalized to that just before the application. NFA $(n=5-9)$, diclofenac $(n=5)$, and indomethacin $(n=5)$ significantly inhibited EAAT1 currents. Aspirin $(n=4)$ did not affect EAAT1 currents. The inhibition by NFA was always more remarkable at $-50 \mathrm{mV}$ than at $-120 \mathrm{mV}$. $* P<0.05$ vs. the control group. ${ }^{\#} P<0.05$ vs. the $-50 \mathrm{mV}$ group. Tukey's test following ANOVA. C: Concentration-response curves of EAAT1 currents at -50 $\mathrm{mV}$ in the absence or the presence of NFA $(\mathrm{n}=9)$ (left) or diclofenac $(\mathrm{n}=8)$ (right). The currents were normalized to the maximal current induced by $3 \mathrm{mM} \mathrm{L}-$ Glu. $\mathrm{I}_{\max }$ and $\mathrm{K}_{0.5}$ were calculated using every concentration-response trace by fitting with the following equation: $\mathrm{I}=\mathrm{I}_{\max }[\mathrm{L}-\mathrm{Glu}] /\left(\mathrm{K}_{0.5}+\right.$ [L-Glu] $)$, using Graphpad PRISM 4 for Windows. Mean $\mathrm{I}_{\max }$ with the drug was normalized to the mean $\mathrm{I}_{\max }$ without drug. Treatment with NFA $(n=9)$ and diclofenac $(n=8)$ resulted in a decrease in the $\mathrm{I}_{\max }$ without affecting the $\mathrm{K}_{0.5}$ (Student's $t$-test). $* P<0.05$ vs. the control group. Tukey's test following ANOVA. 
transient outward current varied by $\mathrm{Cl}^{-}$. Although NFA is reported to inhibit $\mathrm{CaCC}$ (6), the contribution of $\mathrm{CaCC}$ to EAAT1 current may be negligible at -50 and -120 $\mathrm{mV}$ according to the report. Diclofenac $(100 \mu \mathrm{M}-1$ $\mathrm{mM}$ ) inhibited the EAAT1 current dose-dependently. Indomethacin $(100$ and $300 \mu \mathrm{M})$ inhibited the EAAT1 current, but the effects were even weaker. In the case of diclofenac and indomethacin, the strength of the effects at -50 and $-120 \mathrm{mV}$ were almost the same. Aspirin (100 $\mu \mathrm{M}-1 \mathrm{mM}$ ) had no effects. At $-50 \mathrm{mV}$, co-application of NFA with varying doses of L-Glu significantly reduced the maximal current $\left(\mathrm{I}_{\max }\right)$ to $55.1 \pm 1.8 \%$ (Fig. 1C, left). The affinity for L-Glu $\left(\mathrm{K}_{0.5}\right)$ was not affected by NFA (from $50.0 \pm 5.0$ to $33.9 \pm 6.3 \mu \mathrm{M}$ ). Similarly, diclofenac resulted in a decrease in the $\mathrm{I}_{\max }(81.7 \pm 1.2 \%)$ without affecting the $\mathrm{K}_{0.5}$ (from $27.3 \pm 4.0$ to $35.2 \pm 6.3 \mu \mathrm{M}$ ) in a non-competitive manner (Fig. 1C, right). Although vehicle alone had no significant effect on $\mathrm{I}_{\max }$ and $\mathrm{K}_{0.5}$ (data not shown), the $\mathrm{K}_{0.5}$ value for diclofenac-vehicle was smaller than that for NFA-vehicle, which might have been attributed to the slight conformational change caused by DMSO and MeOH. Taken together, these results suggest that NFA and diclofenac modulate EAAT1 via a site different from the L-Glu recognition site.

AA is known to decrease EAAT1 current in a noncompetitive manner (7). Therefore, we compared the effects of NFA and diclofenac when co-applied with AA. The doses used for NFA and diclofenac were determined so as to obtain the equivalent effects and not to reach maximum effects (Fig. S1) (Supplementary Figure: available in the online version only). NFA and AA have an almost additive effect (Fig. 2A). Diclofenac and AA did not show an additive effect (Fig. 2B). The enhancement by AA of diclofenac's effect was significantly weaker than that of NFA's effect (Fig. 2C). These results imply that NFA and diclofenac interact with EAAT1 in different manners.

Figure 3A shows representative current-voltage curves for the EAAT1 currents in the presence or in the absence of NFA. The current value in the presence of NFA alone has been subtracted from that in the presence of NFA and L-Glu. NFA produced a significant leftward shift of reversal potential $\left(\mathrm{E}_{\mathrm{rev}}\right)$ (from $28.9 \pm 5.1$ to $5.2 \pm 5.7 \mathrm{mV}$ ). At the potentials more negative than $-100 \mathrm{mV}$, NFA increased the current amplitude. The influence of NFA on the current amplitude was voltage-dependent (Fig. 3B). Diclofenac inhibited the current amplitude to the same extent at all negative potentials and had no effects on the $\mathrm{E}_{\text {rev }}$ (from $33.8 \pm 9.1$ to $27.5 \pm 6.1 \mathrm{mV}$ ) (Fig. 3: C and $\mathrm{D})$. These results further support the implication that NFA and diclofenac interact with EAAT1 in different manners.

The differences in voltage dependency and the effects

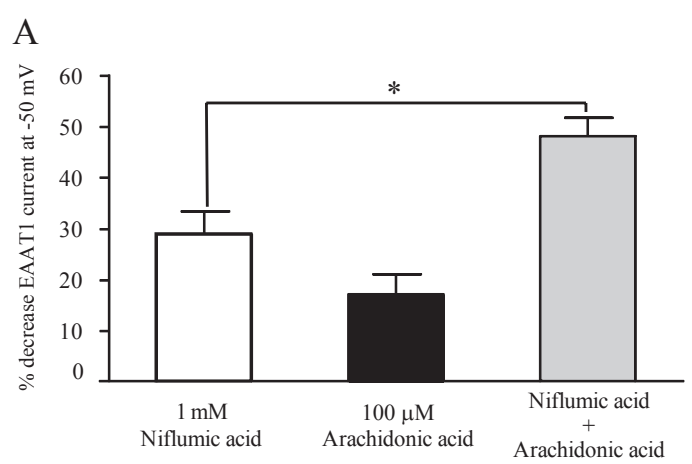

B
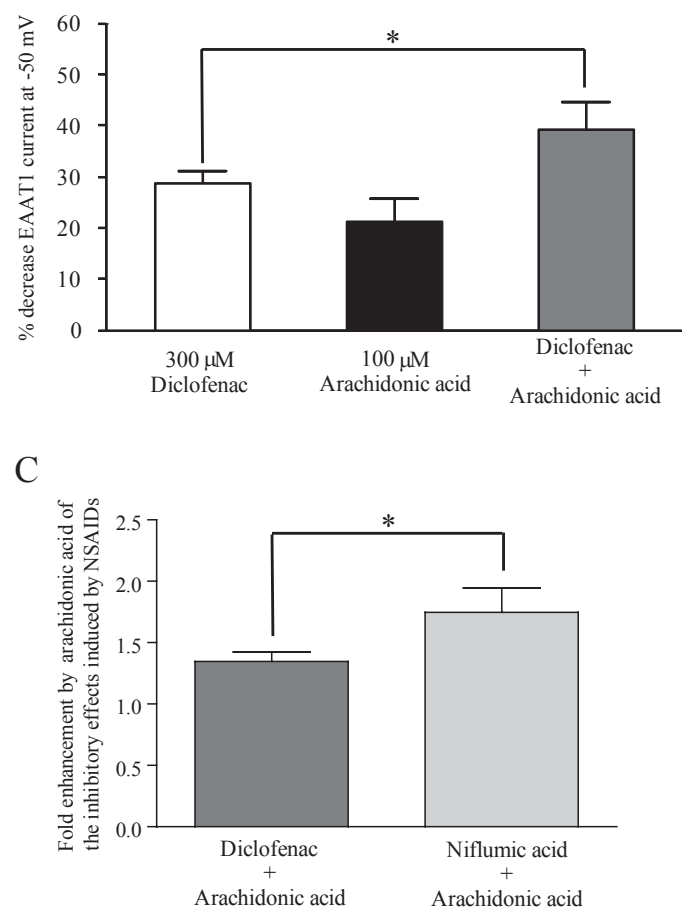

Fig. 2. AA differently influenced the effects of NFA and diclofenac. $\mathrm{A}$ and $\mathrm{B}$ : The percentage decrease was calculated by comparing the current amplitude induced by $30 \mu \mathrm{M} \mathrm{L}-$ Glu with drugs to that induced by L-Glu alone at $-50 \mathrm{mV}$. NFA and AA have an almost additive effect $(28.9 \pm 4.6 \%$ decrease for NFA [ $1 \mathrm{mM}], 17.4 \pm 3.8 \%$ decrease for $\mathrm{AA}[100 \mu \mathrm{M}], 48.3 \pm 3.4 \%$ decrease for $\mathrm{NFA}+\mathrm{AA}, \mathrm{n}=4$ ). Although AA also enhanced the inhibitory effect of diclofenac, the enhancement was even weaker than that of NFA $(28.7 \pm 2.6 \%$ decrease for diclofenac $[300 \mu \mathrm{M}], 21.3 \pm 4.3 \%$ decrease for AA [100 $\mu \mathrm{M}], 39.3 \pm 4.0 \%$ decrease for diclofenac $+\mathrm{AA}, \mathrm{n}=5$ ). $* P<0.05$ vs. the NSAIDs alone group. Paired $t$-test. C: The fold enhancement was calculated by comparing the percentage decrease by NSAIDs to that by NSAIDs with AA at $-50 \mathrm{mV}$. The enhancement by AA of diclofenac's effects was significantly weaker than that of NFA's effect $(1.7 \pm 0.2$ vs. $1.3 \pm 0.1$ fold; $* P<0.05$, Student's $t$-test $)$.

on $\mathrm{E}_{\text {rev }}$ suggest that NFA and diclofenac regulate EAAT1 via different mechanisms. The EAAT1 currents are the net result of charge movements from amino acid (aa) and ion cotransport $\left(\mathrm{Na}^{+} / \mathrm{H}^{+} / \mathrm{K}^{+}\right)\left(\mathrm{I}_{\mathrm{aa}}\right)$ and the ligand-gated $\mathrm{Cl}^{-}$conductance $\left(\mathrm{I}_{\mathrm{Cl}}\right)(8)$. NFA has been reported to en- 
A

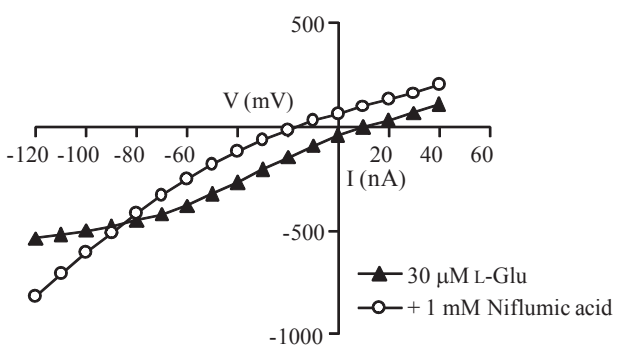

B

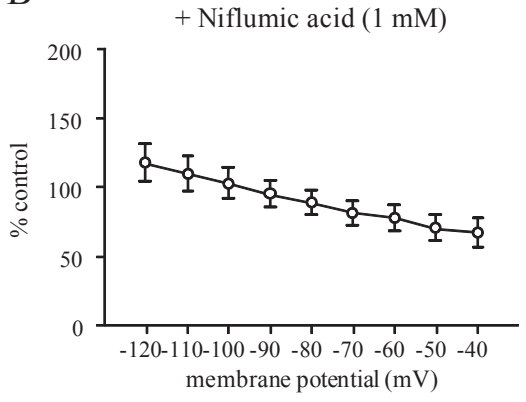

C

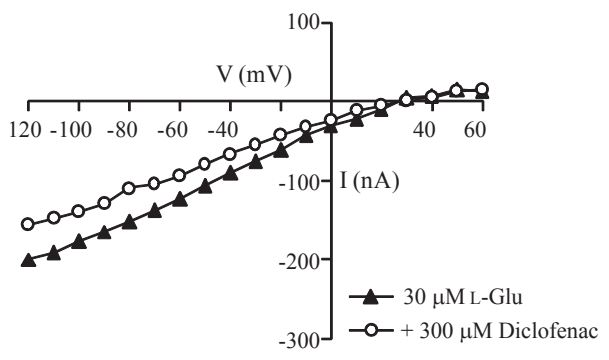

D

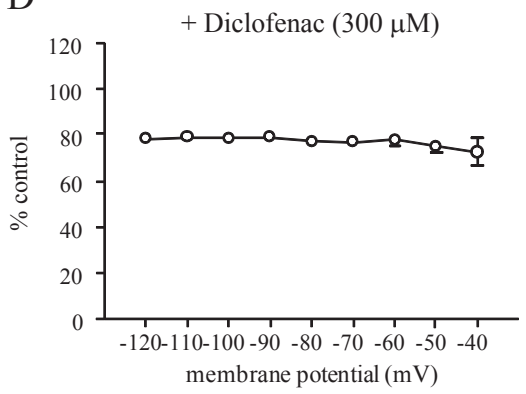

Fig. 3. The effect of NFA is voltage-dependent. A and C: Representative current-voltage relations for $30 \mu \mathrm{M}$ L-Glu in the presence or the absence of $1 \mathrm{mM} \mathrm{NFA} \mathrm{(A)} \mathrm{and} 300 \mu \mathrm{M}$ diclofenac $(\mathrm{C})$. The current-voltage relationships were obtained with a holding potential of $-50 \mathrm{mV}$ and implementing 400-ms voltage jumps in $10-\mathrm{mV}$ increments over the range from -120 to $+60 \mathrm{mV}$. In the control, current values at steady-state have been subtracted from that in the presence of L-Glu. In the drug (NFA or diclofenac)-treated group, the current value in the presence of drug alone has been subtracted from that in the presence of the drug and L-Glu. NFA $(\mathrm{n}=10)$ produced a leftward shift of $\mathrm{E}_{\mathrm{rev}}(P<0.05)$, whereas diclofenac $(\mathrm{n}=4)$ had no effect (paired $t$-test). B and D: The relationship between the effects and the holding potential. The current in the presence of these drugs was normalized to that obtained just before the application. The effect of NFA $(\mathrm{n}=10)$ was voltage-dependent. Diclofenac $(n=5)$ inhibited the current amplitude to the same extent at all negative potentials. hance the L-Glu-induced EAAT4 current by activating an uncoupled $\mathrm{H}^{+}$conductance (5). In our preliminary data, NFA did not cause a modulation in the rate of $I_{a a}$ and $\mathrm{I}_{\mathrm{Cl}}$, but rather modulates an uncoupled $\mathrm{H}^{+}$conductance of EAAT1 (unpublished observation), which may be related to the effect of NFA obtained in our study. In contrast, diclofenac altered only the current amplitude but not the $\mathrm{E}_{\text {rev }} . \mathrm{E}_{\text {rev }}$ for the net current depends on the relative magnitude of $\mathrm{I}_{\mathrm{aa}}$ and $\mathrm{I}_{\mathrm{Cl}}$ (8). Diclofenac might have affected both $\mathrm{I}_{\mathrm{aa}}$ and $\mathrm{I}_{\mathrm{Cl}}$ so as not to change $\mathrm{E}_{\text {rev }}$. The mechanism for the effects of diclofenac needs further investigation.

In our study, the influences on EAAT1 currents were caused by NFA, diclofenac, and indomethacin but not by aspirin. Mefenamic acid, diclofenac, and indomethacin are known to induce reproducible symptoms in patients with affective disorder (9). Aspirin has not been reported to have such side defects to date. If you take into account that GLAST (rodent EAAT1)-deficient mice showed phenotypic abnormalities related to schizophrenia (10), our results suggest that the psychiatric side effects of some NSAIDs have correlation with their effects on L-Glu transporters. Because NSAIDs have high affinity with the plasma protein, their brain delivery is restricted by the blood brain barrier (11). For example, the concentration of enantiomers of ibuprofen in cerebrospinal fluid is less than $1.5 \mu \mathrm{M}$ after therapeutic application (12). Based on these data, the effective concentrations of NFA and diclofenac in our study are higher than that expected for therapeutic application. Habjan et al. recently indi- cated that EAAT1 current was inhibited by NFA. The extent of the effects was application time-dependent and partially irreversible (4), raising the possibility that NFA and diclofenac modulate EAAT1 after long-term administration.

NSAIDs are widely used for patients with inflammation, fever, and pain. Our results may help understanding the mechanisms of side effects caused by some NSAIDs.

\section{Acknowledgments}

We thank Dr. Yasuda-Kamatani and Dr. Shimamoto for providing the cDNA of EAAT1 and Dr. Nakagawa and Dr. Shigeri for helpful suggestions. This work was partly supported by a Grant-in-Aid for Young Scientists from MEXT, Japan (KAKENHI 18700373, 21700422); a Grant for Research on Publicly Essential Drugs and Medical Devices from JHSF; and a Health and Labor Science Research Grant for Research on Risks of Chemicals from MHLW, Japan, awarded to K.S.

\section{References}

1 Beart PM, O'Shea RD. Transporters for L-glutamate: an update on their molecular pharmacology and pathological involvement. Br J Pharmacol. 2007;150:5-17.

2 Wahner AD, Bronstein JM, Bordelon YM, Ritz B. Nonsteroidal anti-inflammatory drugs may protect against Parkinson disease. Neurology. 2007;69:1836-1842.

3 Browning $\mathrm{CH}$. Nonsteroidal anti-inflammatory drugs and severe psychiatric side effects. Int J Psychiatry Med. 1996;26:25-34.

4 Habjan S, Vandenberg RJ. Modulation of glutamate and glycine 
transporters by niflumic, flufenamic and mefenamic acids. Neurochem Res. 2009;34:1738-1747.

5 Poulsen MV, Vandenberg RJ. Niflumic acid modulates uncoupled substrate-gated conductances in the human glutamate transporter EAAT4. J Physiol. 2001;534:159-167.

6 White MM, Aylwin M. Niflumic and flufenamic acids are potent reversible blockers of $\mathrm{Ca}^{2+}$-activated $\mathrm{Cl}^{-}$channels in Xenopus oocytes. Mol Pharmacol. 1990;37:720-724.

7 Zerangue N, Arriza JL, Amara SG, Kavanaugh MP. Differential modulation of human glutamate transporter subtypes by arachidonic acid. J Biol Chem. 1995;270:6433-6435.

8 Wadiche JI, Amara SG, Kavanaugh MP. Ion fluxes associated with excitatory amino acid transport. Neuron. 1995;15:721-728.

9 Jiang HK, Chang DM. Non-steroidal anti-inflammatory drugs with adverse psychiatric reactions: five case reports. Clin Rheumatol. 1999;18:339-345.

10 Karlsson RM, Tanaka K, Saksida LM, Bussey TJ, Heilig M, Holmes A. Assessment of glutamate transporter GLAST (EAAT1)-deficient mice for phenotypes relevant to the negative and executive/cognitive symptoms of schizophrenia. Neuropsychopharmacology. 2009;34:1578-1589.

11 Parepally JM, Mandula H, Smith QR. Brain uptake of nonsteroidal anti-inflammatory drugs: ibuprofen, flurbiprofen, and indomethacin. Pharm Res. 2006;23:873-881.

12 Bannwarth B, Lapicque F, Pehourcq F, Gillet P, Schaeverbeke T, Laborde C, et al. Stereoselective disposition of ibuprofen enantiomers in human cerebrospinal fluid. Br J Clin Pharmacol. 1995;40:266-269. 